\title{
壳聚糖酯钉络合物催化苯乙酮的不对称氢转移反应
}

\author{
刘兵周宏勇李云庆王家喜* \\ (河北工业大学化工学院 天津 300130)
}

\begin{abstract}
摘要 研究了硬脂酸壳聚糖酯 $(O$-stearoyl chitosan, $\mathrm{SCS})$ 与 $\left[\mathrm{RuCl}_{2}(p \text {-cymene })\right]_{2}$ 原位形成催化剂对以异丙醇、甲酸钠为氢 源的苯乙酮不对称氢转移反应的催化性能, 考察了反应时间、温度、四氢呋喃用量等因素对催化反应的影响. 硬脂酸 壳聚糖酯在异丙醇/四氢呋喃的溶液中以胶束形式存在, 平均胶束粒径 $213 \mathrm{~nm}$. 以异丙醇为氢供体时, 产物 1-苯乙醇 $e e$ 值可达 $62.9 \%$, 四氢呋喃的用量对催化活性影响较大. 当异丙醇与四氢呋喃体积比为 $1: 1$ 时催化活性随温度升高而升 高, $100{ }^{\circ} \mathrm{C}$ 时催化反应的催化效率(TOF)值可达 $48.5 \mathrm{~h}^{-1}$; 以甲酸钠作氢源时, 反应的 $\mathrm{TOF}$ 值及 1 -苯乙醇的 $e e$ 分别可达 $29.4 \mathrm{~h}^{-1}$ 及 $53.0 \%$.
\end{abstract}

关键词 壳聚糖; 硬脂酸壳聚糖酯; 不对称氢转移; 苯乙酮

\section{Asymmetric Transfer Hydrogenation of Acetophenone Promoted by Chitosan Ester Ruthenium Complex}

\author{
Liu, Bing Zhou, Hongyong Li, Yunqing Wang, Jiaxi* \\ (School of Chemical Engineering, Hebei University of Technology, Tianjin 300130)
}

\begin{abstract}
The catalytic properties of the catalyst generated in situ from the reaction of $\mathrm{Ru}$ (II) compounds with chitosan based ligands were evaluated in the asymmetric transfer hydrogenation of acetophenone using isopropanol or sodium formate as hydrogen source. The effects of reaction time, temperature and the dosage of THF on the performance of the catalyst were evaluated. The $O$-stearoyl chitosan (SCS) is in the form of micelles with the average size of $213 \mathrm{~nm}$ in $i$-PrOH/THF solution. Using $i$-PrOH as hydrogen source, the ee value of 1-phenylethanol is up to $62.9 \%$. The dosage of THF strongly affected the catalytic activity. The catalytic activity (TOF) is up to $30.4 \mathrm{~h}^{-1}$ at the volume ratio of $i$-PrOH to THF being $1: 1$. The TOF increased with the increase of the temperature. At $100{ }^{\circ} \mathrm{C}$, the TOF is up to $48.5 \mathrm{~h}^{-1}$. While using HCOONa as the hydrogen source, the TOF and ee values of 1-phenylethanol are $29.4 \mathrm{~h}^{-1}$ and $53.0 \%$, respectively.
\end{abstract}

Keywords chitosan; $O$-stearoyl chitosan; asymmetric transfer hydrogenation; acetophenone

光学活性醇是制备手性药物的重要中间体，其选择 性合成具有重要的理论意义及工业应用价值 ${ }^{[1 \sim 3]}$. 过渡 金属络合物催化的不对称氢转移反应制备手性醇具有 反应条件温和, 催化活性高, 操作简单等优点而备受关 注 $^{[4 \sim 6]}$. 手性配体及其金属络合物的结构对催化剂的催 化活性及不对称选择性具有重要影响, 环境友好催化剂 的研究具有重要的意义.

壳聚糖(CS)含有大量可供功能化修饰及与金属配 位的氨基和羟基, 是良好的手性配体原材料. 基于壳聚 糖的不对称催化反应已经引起人们的广泛关注 ${ }^{[7,8]}$. 壳
聚糖参与的非均相苯乙酮氢转移催化反应已有较多研 究 ${ }^{[911]}$, 但将壳聚糖修饰后应用于均相氢转移反应的研 究较少. Babin 等 ${ }^{[2]}$ 将 CS 单环 6-差基酯化合成出可溶性 6-取代特戊酰基壳聚糖酯, 发现其与钓络合物组成的催 化体系对不对称氢转移反应具有较好的催化活性. 为了 进一步探讨壳聚糖上取代基对其参与催化反应的影响, 研究天然手性源在不对称催化反应中的应用前景, 本文 考察了 $O$-硬脂酸壳聚糖酯 $(O$-stearoyl chitosan, SCS $)$ 与 $\left[\mathrm{RuCl}_{2}(p \text {-cymene })\right]_{2}$ 原位形成催化剂对以异丙醇、甲酸钠 为氢源的苯乙酮的氢转移反应的催化性能.

\footnotetext{
*E-mail: wangjiaxi@hebut.edu.cn

Received June 18, 2014; revised July 30, 2014; published online August 26, 2014

Project supported by the Natural Science Foundation of Hebei Province (No. B2011202087).

河北省自然科学基金(No. B2011202087)资助项目.
} 


\section{1 结果与讨论}

\section{1 硬脂酸壳聚糖酯的合成与表征}

壳聚糖由于极性很大, 在有机溶剂中的溶解性很 低. 为了提高其在有机溶剂中的溶解性, 探讨壳聚糖 基手性源化合物参与的不对称反应的性能, 我们参考 文献[13]的方法合成了 6-硬脂酸壳聚糖酯, 反应式如 Scheme 1 所示.
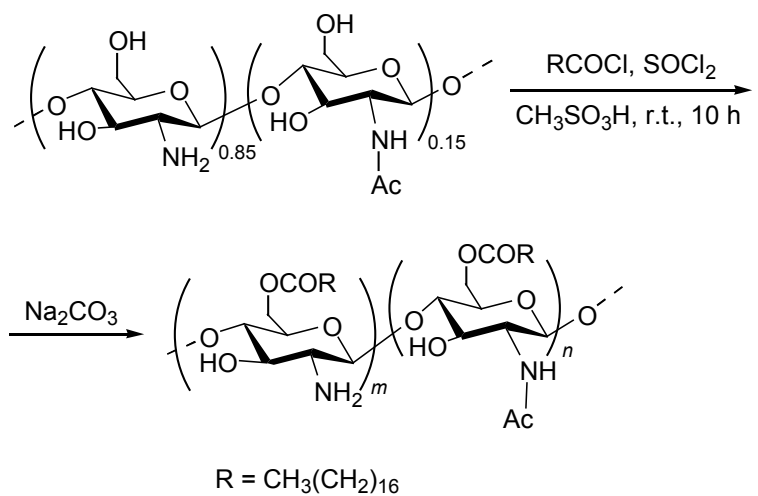

\section{Scheme 1}

所得硬脂酸壳聚糖酯(SCS) 经 FT-IR 及 NMR 分析, $\mathrm{SCS}$ 在 1741,1196 和 $1079 \mathrm{~cm}^{-1}$ 处出现 $\mathrm{C}=\mathrm{O}$ 键和 $\mathrm{C}-\mathrm{O}$ 键的伸缩振动吸收峰, 依据 $1599 \mathrm{~cm}^{-1}$ 处游离 $\mathrm{NH}_{2}$ 特征 峰及没有酰胺 $\left(1640 \sim 1650 \mathrm{~cm}^{-1}\right.$ )的信号判定酰基化主 要发生在糖的 6 位伯羟基上. 硬脂酸壳聚糖酯几乎不溶 于水, 在氯仿中溶解性也不好, SCS 的 ${ }^{1} \mathrm{H}$ NMR 在 $\delta$ $2.70 \sim 5.80$ 处出现很宽的吡喃糖苷环 $\mathrm{C}(2) \sim \mathrm{C}(6)$ 上的质 子峰, $\delta 0.88$ 及 $1.26 \sim 2.32$ 出现硬脂酸的烷基上 $\mathrm{CH}_{3}$ 及 $\mathrm{CH}_{2}$ 质子的吸收峰. 依据烷基与糖环上氢的积分比计 算 ${ }^{[13]}$, 硬脂酸的酰化取代度为 1.0 .

由于异丙醇是后续氢转移反应的氢源及反应溶剂, 而 SCS 在异丙醇中不溶, 为此我们考虑用 $\mathrm{THF} / i-\mathrm{PrOH}$ 为溶剂评价 SCS 参与反应的催化性能. 为了了解 SCS 在 $\mathrm{THF} / i$-PrOH 混合溶剂中的溶解状态, 用动态激光粒 度仪测定了 $\mathrm{SCS}$ 在 $V(\mathrm{THF}): V(i-\mathrm{PrOH})=1: 1$ 混合液中 (浓度为 $0.76 \mathrm{~g} / \mathrm{L}$ ) 的溶解行为, 结果发现 SCS 形成了平 均粒径为 $213 \mathrm{~nm}$, 粒径范围在 50 700 nm 之间的胶束 (图 1). 为了进一步了解四氢呋喃/异丙醇中 SCS 与 $\left[\mathrm{RuCl}_{2}(p \text {-cymene })\right]_{2}$ 络合后的状态, 我们测定了 SCS-Ru 催化剂在 $\mathrm{THF} / i-\mathrm{PrOH}$ 溶液中形成胶束的扫描电镜 $(\mathrm{SEM})$ (图 2). 从图 2 中可以看出, SCS-Ru催化剂聚集成 长度约为 $300 \mathrm{~nm}$, 宽度约为 $100 \mathrm{~nm}$ 的棒状胶束.

\section{2 异丙醇为氢源的苯乙酮氢转移反应}

以 $\mathrm{SCS}$ 与 $\left[\mathrm{RuCl}_{2}(p \text {-cymene })\right]_{2}$ 原位形成的 $\mathrm{Ru}$ 络合物 为催化剂, 异丙醇为氢源, 苯乙酮的氢转移反应选择性

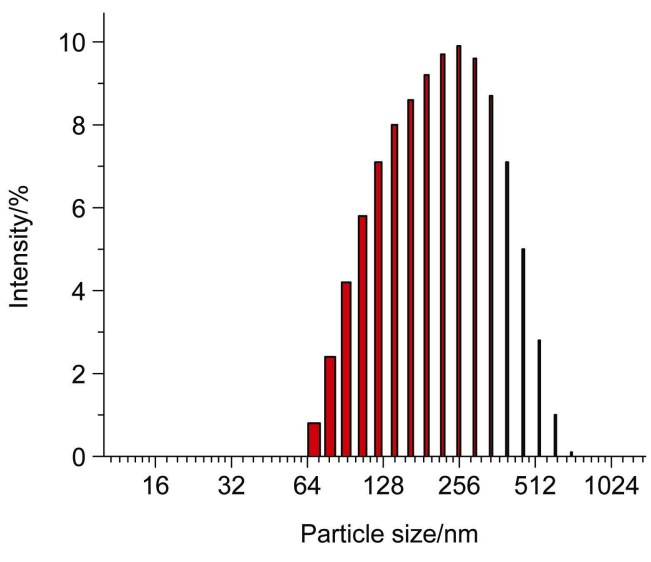

图 $1 \mathrm{SCS}$ 胶束的粒径分布

Figure 1 The particle size distribution of SCS micelle

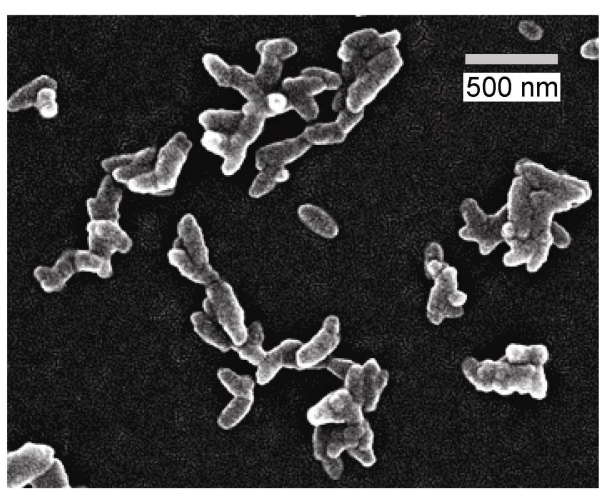

图 $2 \mathrm{SCS}-\mathrm{Ru}$ 催化剂胶束的 SEM 图

Figure 2 The SEM graph of SCS-Ru micelle

形成 $S$-苯乙醇(Eq. 1), 反应结果列于表 1. 从表 1 中可以 看出, 与未加 $\mathrm{SCS}$ 的空白实验相比, $\left[\mathrm{RuCl}_{2} \text { ( } p \text {-cymene) }\right]_{2} /$ SCS 催化体系的催化活性及不对称选择性有了很大的 提高, 产物的 $e e$ 值从 5\%增加到 $60 \%$ (表 1, Entries 1, 2). 催化反应的 TON 随时间几乎线性增加, 而产物的 $e e$ 值 几乎不随反应时间的延长而变化. 与文献[12]报道的特 戊酰基壳聚糖酯配体相比，尽管 ee 值略低, 但催化活性 有了较大的提高.

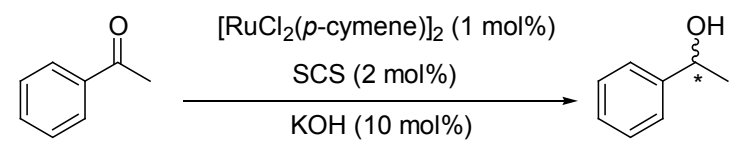

\subsection{1 反应温度的影响}

催化反应的催化活性及不对称诱导效应与反应温 度密切相关, 催化剂的 TON 随反应温度几乎是线性增 加, 而 $e e$ 值随反应温度的升高而下降(图 3), 这一结果符 合不对称催化的一般规律 ${ }^{[14,15]}$. 由 Arrhenius ${ }^{[16]}$ 公式计 算,本催化反应的表观活化能为 $E_{\mathrm{a}}=24.31 \mathrm{~kJ} / \mathrm{mol}$.

\subsection{2 四氢呋喃的影响}

由于 SCS 溶于 THF, 而不溶于异丙醇, THF 的量对 
表 1 以异丙醇为氢源的 $\left[\mathrm{RuCl}_{2}(p \text {-cymene })\right]_{2} / \mathrm{SCS}$ 催化苯乙酮 的不对称氢转移反应 ${ }^{a}$

Table 1 Asymmetric transfer hydrogenation of acetophenone with $\left[\mathrm{RuCl}_{2}(p \text {-cymene) }]_{2} / \mathrm{SCS}\right.$ using $i$-PrOH as hydrogen source

\begin{tabular}{cccc}
\hline Entry & Time $/ \mathrm{h}$ & $e e / \%$ (conf.) & $\mathrm{TOF} / \mathrm{h}^{-1}$ \\
\hline $1^{b}$ & 3 & $1.5(S)$ & 1.2 \\
& 6 & $3.2(S)$ & 0.9 \\
& 9.5 & $5.0(S)$ & 0.9 \\
& 12 & $0.7(S)$ & 0.8 \\
2 & 3 & $60.6(S)$ & 20 \\
& 6 & $59.9(S)$ & 13.4 \\
& 9.5 & $60.0(S)$ & 10.2 \\
& 12 & $60.0(S)$ & 9.4 \\
\hline
\end{tabular}

${ }^{a}$ Reaction conditions: acetophenone $2 \mathrm{mmol}, 25{ }^{\circ} \mathrm{C}, i$-PrOH $6 \mathrm{~mL}$, THF $5 \mathrm{~mL}$; ${ }^{b}$ without ligand.

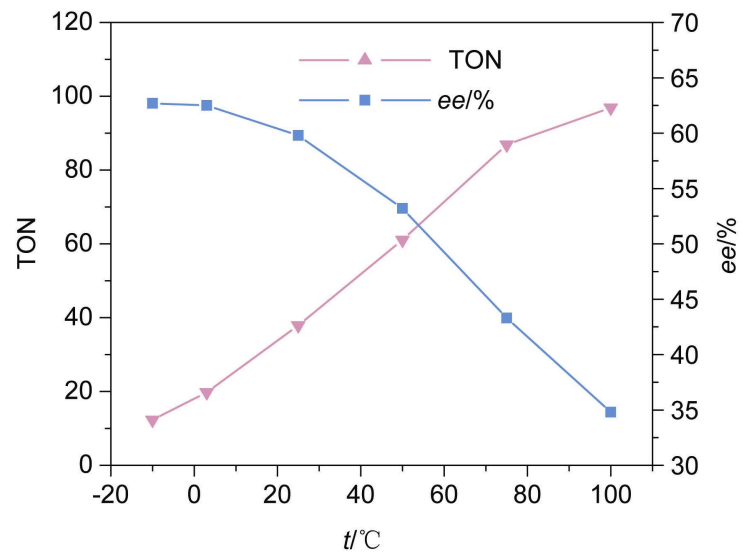

图 3 反应温度对 $\mathrm{TON}$ 和 $e e$ 的影响

Figure 3 Effect of reaction temperature on the TON and ee Acetophenone (1 mmol), $\left[\mathrm{RuCl}_{2}(p \text {-cymene })\right]_{2}(1 \mathrm{~mol} \%)$, SCS $(2 \mathrm{~mol} \%)$, $\mathrm{KOH}(10 \mathrm{~mol} \%), i$-PrOH $(6 \mathrm{~mL})$, THF $(5 \mathrm{~mL}), 2 \mathrm{~h}$

催化剂的溶解状态具有重要的影响, THF 对催化反应的 影响结果见图 4. 从图中可以看出, 反应的 $e e$ 值随 $\mathrm{THF}$ 量的变化较小, 基本保持在 $60 \%$, TON 值随着体系中的 $\mathrm{THF} / i-\mathrm{PrOH}$ 体积比例的增加出现先升高后降低的现象. 当 $\mathrm{THF} / i-\mathrm{PrOH}$ 的体积比为 1 时, 催化的 TON 达到 60.0, 继续增加 THF 用量时, 催化反应活性 TON 值迅速降低, 这一结果可能是由于 SCS 在不同介质中的聚集状态不 同造成的. SCS 由亲油的硬脂酸长链及亲水性的壳聚糖 组成, 在溶液中可以形成不同的团聚结构, 典型的结构 形式如 Scheme 2. 溶剂极性小时, 壳聚糖可能自相作用 形成硬脂酸长链朝外的构型, 此时游离氨基及羟基被烷 基链包裹紧密，与钉络合物结合及催化反应的概率较 低. 增加 THF 量后, 溶剂极性增加, 有利于壳聚糖结构 聚集在胶束的表面，与钉化合物络合形成催化中心; 继 续增加 $\mathrm{THF}$ 的量时, 可能由于硬脂酸的长烷基链的缠 绕及聚集状态的反转, 胶束结构变化 ${ }^{[17,18]}$, 导致壳聚糖 不同结构单元的胺基及羟基与钉络合物进一步配位形
成低活性的钉络合物 ${ }^{[19]}$, 催化活性的降低. 催化性能与 胶束聚集状态的关系非常复杂，进一步的催化性能研究 还在进行中

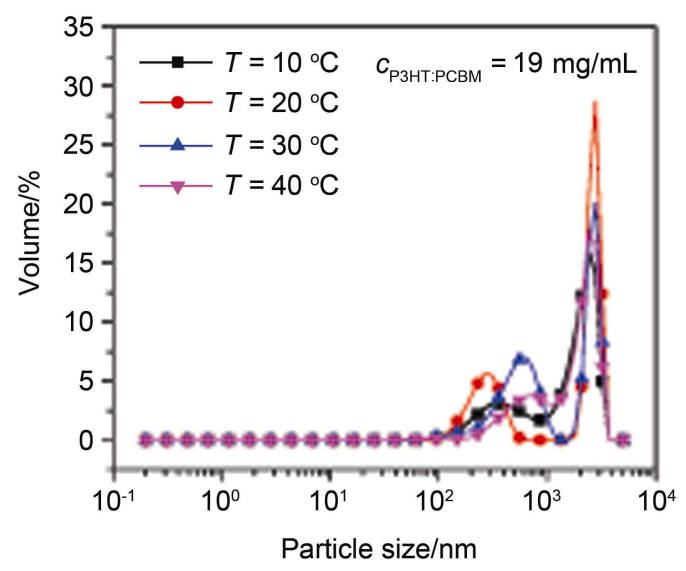

图 4 四氢呋喃/异丙醇比例对 TON 和 $e e$ 的影响

Figure 4 Effect of $V(\mathrm{THF}) / V(i-\mathrm{PrOH})$ on the TON and $e e$ Reaction conditions: acetophenone $(1 \mathrm{mmol}), 25{ }^{\circ} \mathrm{C}, 2 \mathrm{~h},\left[\mathrm{RuCl}_{2}(p\right.$-cymene) $]_{2}(1 \mathrm{~mol} \%)$, SCS (2 mol\%), KOH (10 mol\%)

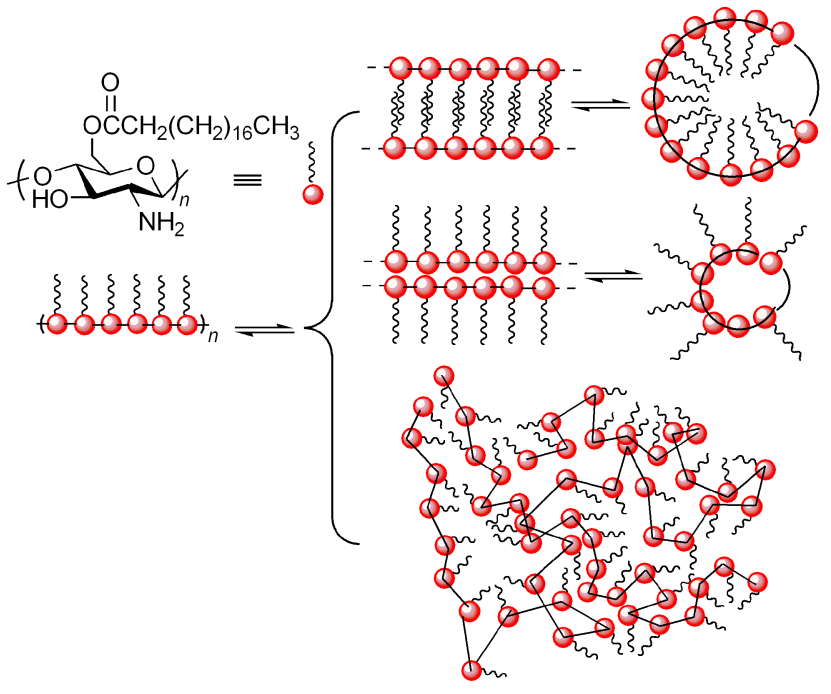

Scheme 2

\section{3 甲酸钠为氢源的苯乙酮氢转移反应}

鉴于 SCS 在异丙醇中的溶解性问题及 SCS 具有一 定表面活性剂的特点, 我们考察了 SCS 对以甲酸钠为氢 源的氢转移反应的催化性能(Eq. 2). 在催化剂的作用下, 甲酸钠还原苯乙酮形成 $S$ 型为主的苯乙醇, 反应结果列 于表 2 中. 从表中可以看出, 催化剂的催化活性随温度 升高而升高, $65{ }^{\circ} \mathrm{C}$ 时 TOF 值达到最大值 29.4; 随后催化 活性随温度的升高而下降. 苯乙醇的 $e e$ 值随温度的升 高先增大, $50{ }^{\circ} \mathrm{C}$ 时苯乙醇的 $e e$ 值达到最大值 $57.3 \%$; 随 后也随温度的增加而下降. 值得注意的是本研究体系没 有出现水的抑制效应 ${ }^{[20]}$. 
<smiles>CC(=O)c1ccccc1</smiles>

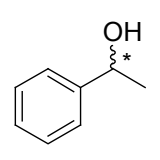

表 2 以甲酸钠为氢源的 $\left[\mathrm{RuCl}_{2}(p \text {-cymene })\right]_{2} / \mathrm{SCS}$ 催化苯乙酮 的不对称氢转移反应

Table 2 Asymmetric transfer hydrogenation of acetophenone with $\left[\mathrm{RuCl}_{2}(p \text {-cymene })\right]_{2} / \mathrm{SCS}$ using $\mathrm{HCOONa}$ as hydrogen source

\begin{tabular}{ccccc}
\hline Entry & Temp. $/{ }^{\circ} \mathrm{C}$ & Time $/ \mathrm{h}$ & $e e^{\circ} \%$ & $\mathrm{TOF} / \mathrm{h}^{-1}$ \\
\hline 1 & 0 & 5 & $49.0(S)$ & 0.04 \\
2 & 25 & 5 & $54.4(S)$ & 0.8 \\
3 & 50 & 5 & $57.4(S)$ & 6.9 \\
4 & 60 & 5 & $54.7(S)$ & 24.5 \\
5 & 65 & 5 & $53.0(S)$ & 29.5 \\
6 & 70 & 5 & $51.0(S)$ & 26.5 \\
7 & 75 & 5 & $50.4(S)$ & 26.0 \\
8 & 100 & 5 & $42.7(S)$ & 11.7 \\
9 & 120 & 5 & $38.8(S)$ & 11.4 \\
10 & 140 & 5 & $35.3(S)$ & 8.6 \\
\hline
\end{tabular}

\section{2 结论}

硬脂酸壳聚糖酯 SCS 在 $\mathrm{THF} / i-\mathrm{PrOH}$ 中形成具有一 定旋光性的胶束, 与钉化合物原位形成 Ru-SCS 催化剂 对苯乙酮的氢转移反应具有较好的催化活性, 形成以 $S$ 构型为主的 1-苯乙醇产物. 在 $\mathrm{THF} / i \mathrm{-PrOH}$ 体系中,催化 反应产物的 $e e$ 值可达 $62.7 \%, e e$ 值几乎不随反应时间的 延长而变化; 催化反应的 $\mathrm{TOF}$ 值随温度升高而升高, $100{ }^{\circ} \mathrm{C}$ 反应 $2 \mathrm{~h}$ 时 TOF 值可达 $48.5 \mathrm{~h}^{-1}$, 产物的 $e e$ 值为 $34.8 \%$. THF 的量对催化活性影响很大. 在 $\mathrm{THF} / \mathrm{H}_{2} \mathrm{O}$ 两 相体系中, 甲酸钠能还原苯乙酮形成 $S$ 构型为主的 1-苯 乙醇产物. TOF 值随反应温度升高呈先增加后降低的趋 势, 在 $65{ }^{\circ} \mathrm{C}$ 时达到最大值 $29.4 \mathrm{~h}^{-1}$; 产物的 $e e$ 值随反应 温度升高也呈现增加后降低的趋势, 在 $50{ }^{\circ} \mathrm{C}$ 时达到最 大值 $57.3 \%$

\section{3 实验部分}

\section{1 仪器与试剂}

红外光谱仪(TENSOR27, 德国布鲁克公司); 核磁 共振谱仪(AVANCE400, 德国布鲁克公司); 气相色谱仪 [SP-6890, 山东鲁南瑞虹化工仪器有限公司, CP-Chirasil-DEX CB 石英柱, 汽化室 $240{ }^{\circ} \mathrm{C}$, 柱温 $130{ }^{\circ} \mathrm{C}$, 检测 器 $240{ }^{\circ} \mathrm{C}$, 载气流速 $1 \mathrm{~mL} / \mathrm{min},(R)$-1-苯乙醇及 $(S)$-1-苯 乙醇保留时间分别为 5.2 及 $5.8 \mathrm{~min}$ ); 激光粒度仪(ZS90, 英国马尔文公司); 旋光仪(WZZ-2S, 上海精密科学仪器 有限公司); 扫描电子显微镜(Hitachi S-4800, 日本日立 公司).
壳聚糖(CS)，河南金润食品添加剂有限公司，粘均 分子量 $5.4 \times 10^{5}$, 脱乙酰度 $85 \%$; 硬脂酸, 天津市化学 试剂一厂; $\left[\mathrm{RuCl}_{2}(p \text {-cymene })\right]_{2}$, Alfa Aesar 公司; 苯乙酮, 国药集团化学试剂有限公司; 甲基磺酸、氯化亚砜、异 丙醇等其它药品均为分析纯.

\section{2 实验方法}

\subsubsection{6-硬脂酸壳聚糖酯的合成}

参考文献[13]合成: 取 $0.85 \mathrm{~g} \mathrm{CS}$ 溶于 $10 \mathrm{~mL}$ 甲基磺 酸及 $2 \mathrm{~mL} \mathrm{SOCl}_{2}$ 的混合液中, 室温摚拌下缓慢滴加 1.8 $\mathrm{mL}$ 硬脂酰氯, 反应 $10 \mathrm{~h}$. 在 $0{ }^{\circ} \mathrm{C}$ 下加入冰水稀释, 硬脂 酸壳聚糖酯析出, 过滤. 粗产物置于 $200 \mathrm{~mL}$ 蒸馏水中, 用 $\mathrm{Na}_{2} \mathrm{CO}_{3}$ 溶液调节至中性，过滤，蒸馏水洗涤二次后， $50{ }^{\circ} \mathrm{C}$ 下真空干燥, 得硬脂酸壳聚糖酯 SCS $(2.3 \mathrm{~g}$, 收率 为 $88 \%) .[\alpha]_{\mathrm{D}}^{20}+3.67$ (c 0.55 , THF); ${ }^{1} \mathrm{H}$ NMR $\left(\mathrm{CDCl}_{3}\right.$, $400 \mathrm{MHz}) \delta: 0.88$ (br s, 3H, $\left.\mathrm{CH}_{3}\right), 1.26[\mathrm{br} \mathrm{s}, 30 \mathrm{H}$, $\left.\left(\mathrm{CH}_{2}\right)_{15}\right], 2.32$ (br s, $\left.2 \mathrm{H}, \mathrm{CH}_{2}\right), 2.79$ (br s, $0.89 \mathrm{H}, \mathrm{H}-2$ of GlcN), $3.20 \sim 5.50$ (br m, 10H, H-1,3,4,5,6 of GlcN); IR (KBr) v: 3444 (m), 2923 (s), 2852 (s), 1740 (s), 1599 (w), 1468 (m), 1379 (w), 1196 (s), 1070 (s), 790 (w), 721 (w), $563(\mathrm{w}) \mathrm{cm}^{-1}$.

\subsection{2 异丙醇为氢源的氢转移反应}

在氮气保护下，向 $100 \mathrm{~mL}$ 厚壁 Schlenk 瓶中加入一 定量 $\left[\mathrm{RuCl}_{2}(p \text {-cymene })\right]_{2} 、 \mathrm{SCS}$ 、苯乙酮、 $i$-PrOH、THF, 混合体系经真空脱气及充高纯氮气 3 次, 在氮气保护下 加入定量 $\mathrm{KOH}$ 的 $i-\mathrm{PrOH}$ 的溶液, 再经真空脱气及充高 纯氮气 3 次后在氮气保护下密闭. 在一定温度下, 反应 一定时间后冷却，溶液经硅胶柱过滤，脱去金属化合物 后用气相色谱检测分析，与 $(R)-1$-苯乙醇及 $(S)$-1-苯乙醇 标样对比, 计算催化效率和产物的 $e e$ 值.

\subsection{3甲酸钠为氢源的氢转移反应}

在氮气保护下，向 $100 \mathrm{~mL}$ 厚壁 Schlenk 瓶中加入定 量 $\left[\mathrm{RuCl}_{2}(p \text {-cymene })\right]_{2} 、 \mathrm{SCS}$ 、苯乙酮、THF，混合体系 经真空脱气及充高纯氮气 3 次, 在氮气保护下加入定量 $\mathrm{HCOONa}$ 的水溶液, 混合体系再经真空脱气及充高纯 氮气 3 次后在氮气保护下密闭. 在一定温度下，反应一 定时间后冷却，溶液经硅胶柱过滤，脱去金属化合物后 用气相色谱检测分析，与 $(R)$-1-苯乙醇及 $(S)$-1-苯乙醇标 样对比，计算催化效率和产物的 $e e$ 值.

\section{References}

[1] Li, X.-N.; Zhou, H.-Y.; Feng, L.; Duan, K.; Wang J.-X. Appl. Organomet. Chem. 2012, 26, 168.

[2] Malacea, R.; Poli, R.; Manoury, E. Coord. Chem. Rev. 2010, 254, 729.

[3] Song, S-S.; Zhou, H.-Y.; Li, X.-N.; Wang, L.-H.; Li, Y.-Q.; Wang, J.-X. Chin. J. Org. Chem. 2014, 34, 706 (in Chinese). 
(宋莎莎, 周宏勇, 李小娜, 王丽华, 李云庆, 王家喜, 有机化学, 2014, 34, 706.)

[4] Fu, Q.; Zhang, L.; Yi, T.; Zou M.-J.; Wang, X.-Y.; Fu, H.-Y.; Li, R.-X.; Chen, H. Inorg. Chem. Commun. 2013, 38, 28.

[5] Li, X.-N.; Zhang, P.-L.; Duan, K.; Wang, J.-X. Chin. J. Org. Chem. 2012, 32, 19 (in Chinese).

(李小娜, 张鹏亮, 段凯, 王家喜, 有机化学, 2012, 32, 19.)

[6] Thakur, K. G.; Ganapathy, D.; Sekar, G. Chem. Commun. 2011, 47, 5076.

[7] Zhao, D.-Q.; He, M.; Zhang, Y.-H.; Huang, M.-Y.; Jiang, Y.-Y. Polym. Adv. Technol. 2003, 14(3 5), 287.

[8] Yi, Y.; Wang, Y.-T.; Wan, L.-L.; Zhang, X.-Y.; Ma, S.-L. J. Wuhan Technol. (Mater. Sci.Ed.) 2007, 22(1), 156.

[9] Yin, M.-Y.; Yuan, G.-L.; Wu, Y.-Q.; Huang, M.-Y.; Jiang, Y.-Y. J. Mol. Catal. A: Chem. 1999, 147, 93.

[10] Zhou, D.-Q.; Zhou, D.-J.; Cui, X.-H.; Wang, F.-M.; Huang, M.-Y.; Jiang, Y.-Y. Polym. Adv. Technol. 2004, 15, 350.

[11] Wei, W.-L.; Hao, S.-J.; Zhou, J.; Huang, M. Y.; Jiang, Y.-Y. Polym. Adv. Technol. 2004, 15, 287.

[12] Babin, M.; Clément, R.; Gagnon, J.; Fontaine, F. G. New J. Chem. 2012, 36, 1548.

[13] Badawy, M. E. I.; Rabea, E. I.; Rogge, T. M.; Stevens, C. V.; Smagghe, G.; Steurbaut, W.; Höfte, M. Biomacromolecules 2004, 5, 589.

[14] Sun, Y.-X.; Guo, Y.; Lu, G.-Z.; Wu, X.-H.; Guo, Y.- L.; Wang, Y.-S.;
Liu, X.-H.; Zhang, Z.-G. J. Mol. Catal. (China) 2004, 18(6), 456 (in Chinese).

(孙延喜, 郭耘, 卢冠忠, 吴小华, 郭杨龙, 王筠松, 刘晓轫, 张 志刚, 分子催化, 2004, 18(6), 456.)

[15] Ding, K.-L.; Fan, Q.-H. Asymmetric Catalysis: New Concepts and Methods, Chemical Industry Press, Beijin, 2009 (in Chinese).

(丁奎岭，范青华，不对称催化新概念与新方法，化学工业出版 社, 北京, 2009.)

[16] Luo, Y.-R.; Yu, S.-Q.; Zhang, D.-Z.; Yao, T.-Y.; Gao, P.-L. Univ. Chem. 2010, 25(3), 35 (in Chinese).

(罗渝然，俞书勤，张祖德，姚天扬，高盘良，大学化学，2010, 25(3), 35.)

[17] Fehring, V.; Kadyrov, R.; Ludwig, M.; Holz, J.; Haage, K.; Selke, R. J. Organomet. Chem. 2001, 621, 120.

[18] Li, J.-H.; Tang, Y.-F.; Wang, Q.-W.; Li, X.-F.; Cun, L.-F.; Zhang, X.-M.; Zhu, J.; Li, L.-C.; Deng, J.-G. J. Am. Chem. Soc. 2012, 134, 18522 .

[19] Watts, C.-C.; Thoniyot, P.; Cappuccio, F.; Verhagen, J.; Gallagher, B.; Singgraram, B. Tetrahedron: Asymmetry 2006, 17, 1301.

[20] Sun, Y.-X.; Guo, Y.; Wu, X.-H.; Meng, X.-L.; Lu, G.-Z.; Guo, Y.-L. Chin. J. Appl. Chem. 2006, 23(1), 42 (in Chinese).

(孙延喜, 郭耘, 吴小华, 孟宪乐, 卢冠忠, 应用化学, 2006, 23(1), 42.)

(Li, L.; Fan, Y.) 